\title{
Note: A sample holder design for sensitive magnetic measurements at high temperatures in a magnetic properties measurement system
}

\author{
A. Arauzo, ${ }^{1, a)}$ E. Guerrero, ${ }^{1}$ A. Urtizberea, ${ }^{1}$ J. Stankiewicz, ${ }^{2}$ and C. Rillo ${ }^{1,2}$ \\ ${ }^{1}$ Servicio de Medidas Físicas, Universidad de Zaragoza, Zaragoza 50009, Spain \\ ${ }^{2}$ Instituto de Ciencia de Materiales de Aragón and Departamento de Física de la Materia Condensada, \\ CSIC-Universidad de Zaragoza, 50009 Zaragoza, Spain
}

(Received 16 April 2012; accepted 30 May 2012; published online 15 June 2012)

\begin{abstract}
A sample holder design for high temperature measurements in a commercial MPMS SQUID magnetometer from Quantum Design is presented. It fulfills the requirements for the simultaneous use of the oven and reciprocating sample option (RSO) options, thus allowing sensitive magnetic measurements up to $800 \mathrm{~K}$. Alternating current susceptibility can also be measured, since the holder does not induce any phase shift relative to the ac driven field. It is easily fabricated by twisting Constantan $($ ) wires into a braid nesting the sample inside. This design ensures that the sample be placed tightly into a tough holder with its orientation fixed, and prevents any sample displacement during the fast movements of the RSO transport, up to high temperatures. (C) 2012 American Institute of Physics. [http://dx.doi.org/10.1063/1.4729380]
\end{abstract}

The magnetic properties measurement system (MPMS) from Quantum Design Inc. ${ }^{1}$ is a SQUID (superconducting quantum interference device) based commercial magnetometer capable of measuring magnetic moments with a high sensitivity of the order of $1 \times 10^{-7} \mathrm{emu}$. The reciprocating sample option (RSO) improves the sensitivity to approximately 5 $\times 10^{-9}$ emu. $^{2,3}$ The oven option extends the temperature range up to $800 \mathrm{~K}^{4}$ Combining the RSO and oven options allows one to measure very weak magnetic moments up to $800 \mathrm{~K}$, such as, for example, found in magnetic phase transitions of thin magnetic films or in diluted systems.

A sample holder suitable for magnetic measurements has to fulfill some requirements, especially with the oven and the RSO options installed. As a general rule, the holder contribution must be minimized by choosing materials with low magnetic susceptibility, keeping the mass of the holder to a minimum. In addition, it should be homogeneous (mass constant over the length) and long enough to extend well beyond the detection coils. With the oven option installed, the holder diameter becomes limited since the oven sample space has a $3.5 \mathrm{~mm}$ inner diameter. ${ }^{4,5}$ Furthermore, the holder must be mechanically and thermally stable up to $800 \mathrm{~K}$. Designs suitable for measurements with the oven option have already been reported. ${ }^{6-8}$ However, to our knowledge, there is no report on a holder specifically designed for measurements with the oven and the RSO options, simultaneously. It is interesting to note that due to the fast movements of the RSO transport, secure sample mounting is more challenging than in most other applications, in order to prevent sample rattling or even slipping during the measurement.

The sample holder presented here meets the above criteria. It is easily fabricated and, therefore, it can be used as a disposable holder thus avoiding any sample contamination. Our design is suitable for solid samples, particularly to

a)Electronic mail: aarauzo@unizar.es. support thin films, since sample orientation can be fixed in the holder, allowing in plane and out of plane magnetic characterization.

The sample holder construction using a wire twister tool of dedicated design is shown in Fig. 1. Four Constantan (c) wires of a $0.25 \mathrm{~mm}$ diameter are tightly twisted into a braid for $4 \mathrm{~cm}$. The sample is then placed in the desirable orientation twisting the wires tightly around it. The wires are twisted for $12 \mathrm{~cm}$ more, since the holder length is limited to $16 \mathrm{~cm}$ when both the RSO and oven options are used. The whole construction, with the sample embedded inside, is mechanically stable, tough and hard, easy to install into the oven.

It is worth mentioning that the sample is tightly held thus avoiding noise in the measurements from loosely mounting. Also, no glue is required to fix the sample. Since the four wires completely wraps the sample, there is not any empty sample space and the holder has a homogeneous distribution of Constantan all along its length. This design minimizes the magnetic moment contribution of the missing Constantan material in the hole. Moreover, since the wire diameter is $0.25 \mathrm{~mm}$, the available sample space in the oven chamber can be used to maximum.

The wire was purchased from Goodfellow. ${ }^{9}$ It is a Constantan Cu55/Ni45 alloy with a composition of Fe $2500 \mathrm{ppm}$, Mn $7500 \mathrm{ppm}, \mathrm{Ni}$ 45\%, and $\mathrm{Cu}$ to balance. The melting point $1498-1573 \mathrm{~K}$ is nearly twice the maximum measurement temperature of $800 \mathrm{~K}$. The thermal expansion coefficient is $14.9 \times 10^{-6} \mathrm{~K}^{-1}$ (at 293-373 K). For a temperature range from 300 to $800 \mathrm{~K}$ this corresponds to a sample displacement of less than $0.5 \mathrm{~mm}$. Magnetic properties of Constantan were characterized measuring the magnetic susceptibility of a bundle of wires. It is a paramagnetic material at oven working temperatures with Curie constant, $C=1.95 \times 10^{-3} \pm 1.6$ $\times 10^{-5} \mathrm{emu} \mathrm{K} / \mathrm{Oe} \mathrm{g}$.

In order to characterize the holder performance three samples were measured: a solid piece of nickel $(9.24 \mathrm{mg})$, a powder $\mathrm{Dy}_{2} \mathrm{O}_{3}$ shaped in a cylinder and a nickel thin film. 


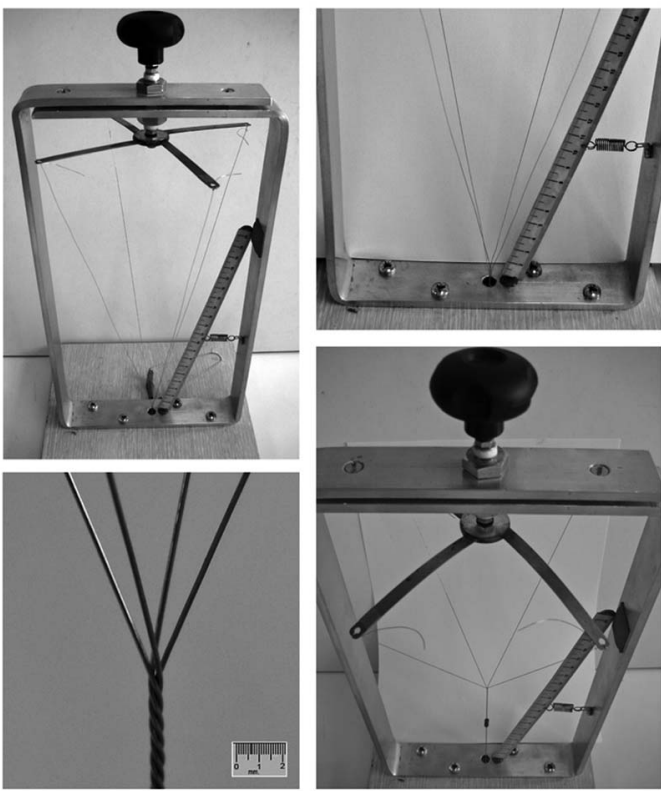

FIG. 1. Sample holder construction nesting the sample inside. The rule at the right side is used for positioning of sample at $4 \mathrm{~cm}$.

They are shown in Fig. 2. The solid piece of nickel was purchased from Alfa Aesar. ${ }^{10}$ The $\mathrm{Dy}_{2} \mathrm{O}_{3}$ sample was prepared by mixing $\mathrm{Dy}_{2} \mathrm{O}_{3}$ powder with a water based ZircarC cement shaping it in the form of a cylinder with a length $l=7 \mathrm{~mm}$ and a diameter $\phi=3 \mathrm{~mm}$. The nickel thin film is $100 \mathrm{~nm}$ thick and was sputter deposited onto a quartz substrate with an area of $1.7 \times 3 \mathrm{~mm}^{2}$ (estimated mass $4.5 \mu \mathrm{g}$ ). The oven thermometer
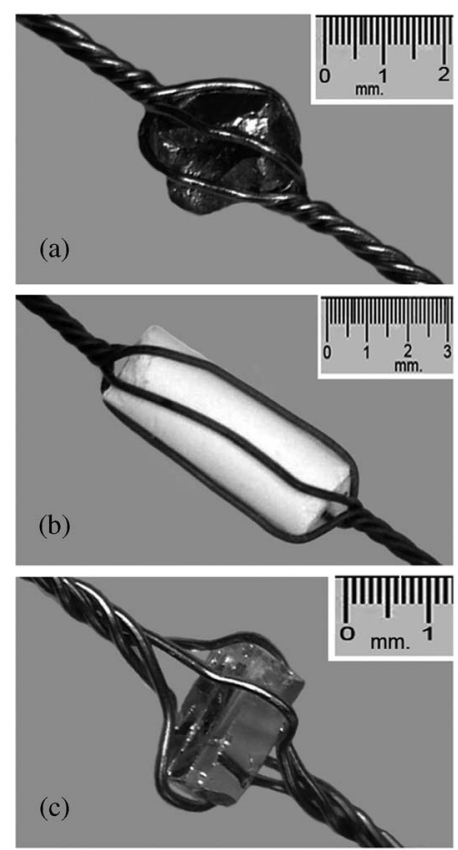

FIG. 2. Three samples installed in the holder. (a) Solid piece of nickel. (b) $\mathrm{Dy}_{2} \mathrm{O}_{3}$ powder shaped to a $7 \mathrm{~mm} \times 2.5 \mathrm{~mm}$ cylinder. (c) Nickel thin film (about $100 \mathrm{~nm}$ ) sputter deposited on a $1.7 \times 3 \mathrm{~mm}^{2}$ quartz surface, mounted perpendicular to the measurement field.

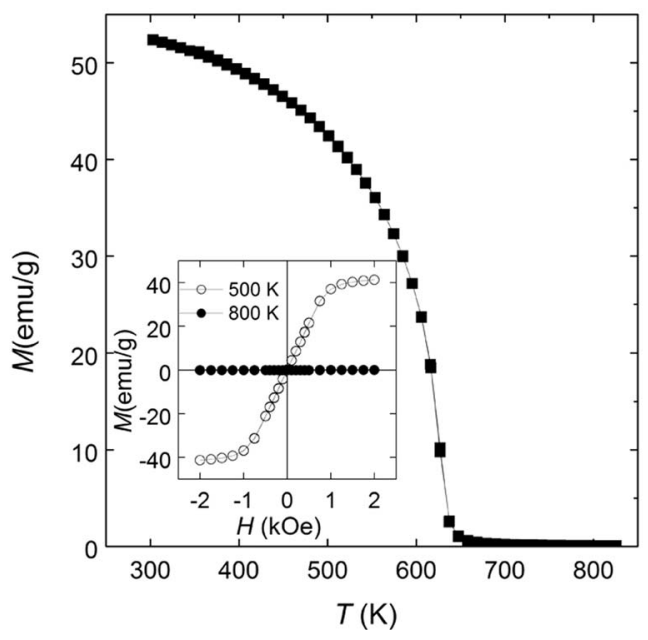

FIG. 3. Magnetization vs. temperature of the nickel bulk sample measured at $H=2 \mathrm{kOe}$. Inset: magnetization vs. magnetic field at $500 \mathrm{~K}$ and $800 \mathrm{~K}$.

was calibrated in the whole temperature range previous to the experimental measurements using a thermocouple.

The ferromagnetic to paramagnetic transition of the nickel bulk sample, shown in Figs. 3 and 4, was measured in order to characterize the temperature response of the holder. The magnetization curve vs. temperature at $H=2 \mathrm{kOe}$ is shown in Fig. 3. The inset displays the magnetization curves showing that at $800 \mathrm{~K}$ the sample behaves as a paramagnet while at $500 \mathrm{~K}$ is ferromagnetic. The Curie temperature $T_{\mathrm{c}}$ $=631.42 \pm 0.4 \mathrm{~K}$, estimated using the reciprocal ac susceptibility data shown in Fig. 4, is in very good agreement with values reported in the literature. ${ }^{11}$

The ac susceptibility of the paramagnetic $\mathrm{Dy}_{2} \mathrm{O}_{3}$ was measured at 200,600,800, and $1000 \mathrm{~Hz}$, to determine the phase shift induced by the holder relative to the ac driven field. The experiments were performed from 300 to $400 \mathrm{~K}$ without the Oven option since its metallic chamber induces a background phase shift (eddy currents), which increases linearly with the frequency, of about $2^{\circ}-3^{\circ}$ at $1 \mathrm{kHz}$. The phase

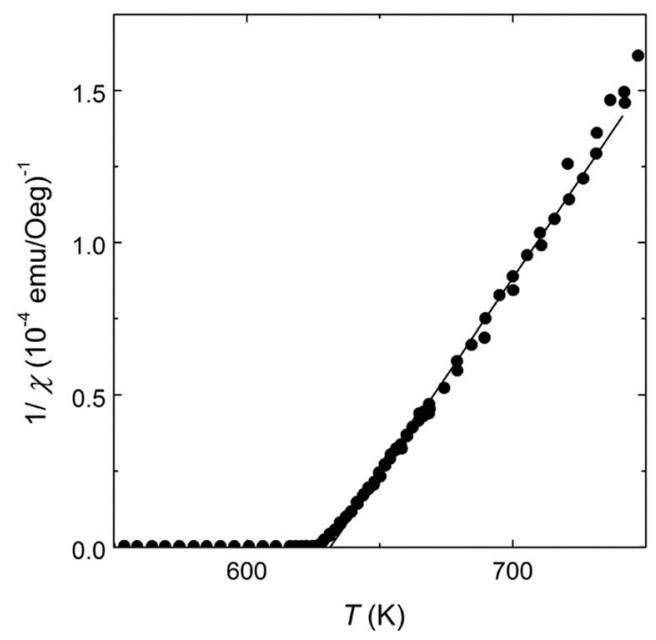

FIG. 4. Reciprocal ac susceptibility of the nickel bulk sample showing evidence of the ferromagnetic to paramagnetic transition at $T_{\mathrm{c}}=631.42 \pm 0.4 \mathrm{~K}$. The solid line represents the extrapolation of the Curie-Weiss law characteristic of the paramagnetic phase. 


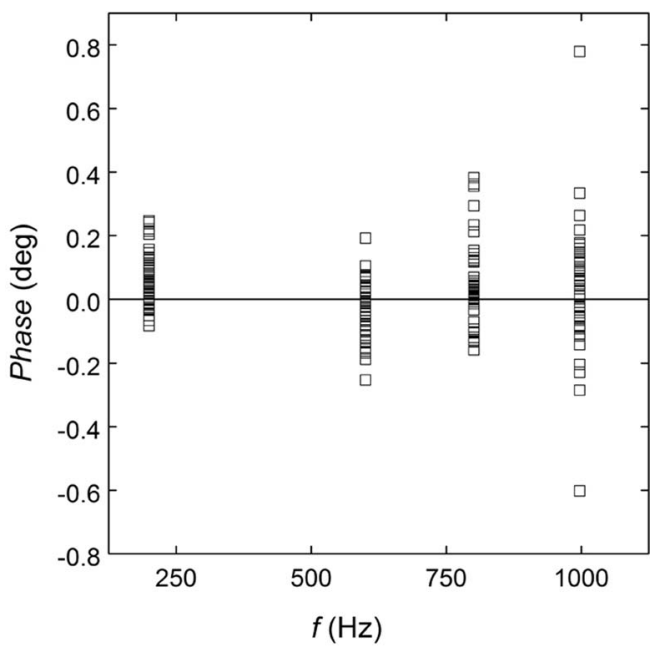

FIG. 5. Alternating current susceptibility phase of the $\mathrm{Dy}_{2} \mathrm{O}_{3}$ measured from 300 to $400 \mathrm{~K}$ without the oven sample space. The holder does not induce any phase shift relative to the ac drive field applied.

shift of the $\mathrm{Dy}_{2} \mathrm{O}_{3}$, shown in Fig. 5, is negligible within the experimental resolution. ${ }^{12}$ Therefore, our holder does not induce any additional phase shift relative to the ac drive field applied.

Finally, a nickel thin film was measured in order to test the suitability of the holder for measurements requiring the highest sensibility. The thin film was fixed in the holder perpendicular to the direction of the magnetic field. Figure 6 shows the magnetic moment, $m$, as a function of the applied field at $300 \mathrm{~K}$ and versus temperature at $2 \mathrm{kOe}$ (Fig. 6 inset). The empty holder shows a weak positive moment of 1.4 $\times 10^{-6} \mathrm{emu}$ at $1 \mathrm{kOe}$ varying linearly with the field. This feature can be assigned to the magnetic moment of a small excess of $0.18 \mathrm{mg}$ of Constantan at the sample space due to incomplete compensation of the wires. Magnetic signal from the sample holder is therefore negligible as compared with sample signal, $m=1.2 \times 10^{-4} \mathrm{emu}$ at $1 \mathrm{kOe}$. Given that the weak magnetic moment of this thin film requires the sensitivity provided by the RSO option, we can affirm that the sample holder design is suitable for the most sensitive magnetic measurements.

A design of sample holder for measurements in a MPMS with the RSO and Oven options has been presented. As compared with previous holder designs, it shows two advantages: (i) it is apt for measurements of very weak magnetic moments

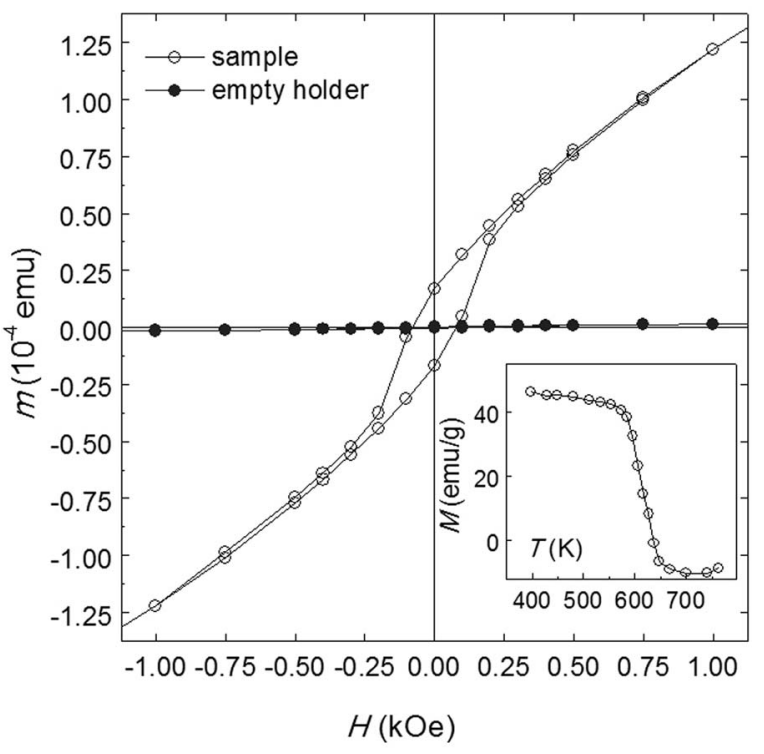

FIG. 6. Magnetic moment at $300 \mathrm{~K}$ of the nickel thin film and empty sample holder. Inset: magnetic moment vs temperature at $2 \mathrm{kOe}$ of the nickel thin film showing the ferromagnetic to paramagnetic transition at $T \mathrm{c} \sim 630 \mathrm{~K}$.

(RSO option) up to $800 \mathrm{~K}$ and, (ii) it fixes sample orientation (thin films). The design proposed is as well suitable for ac measurements since it does not induce any additional phase shift. This design allows high-temperature measurements for different kind of solid samples with diameters up to the maximum available in the oven chamber space.

A.U. acknowledges financial support PTA2010-3639-I and PTA31/11 of her contract from the PTA-MICINN 2010 and PTA-DGA 2011 Programs, respectively.

${ }^{1}$ Quantum Design, Inc. 6325 Lusk Boulevard, San Diego, CA 92121-3733. ${ }^{2}$ Quantum Design, MPMS Reciprocating Sample Option User's Manual.

${ }^{3}$ Quantum Design, MPMS Application Note 1014-822.

${ }^{4}$ Quantum Design, MPMS Sample Space Oven Option User's Manual.

${ }^{5}$ Quantum Design, MPMS Application Note 1014-201.

${ }^{6}$ L. H. Lewis and K. M. Bussmann, Rev. Sci. Instrum. 67, 3537 (1996).

${ }^{7}$ S. Bedanta, O. Petracic, M. Aderholz, and W. Kleemann, Rev. Sci. Instrum. 76, 083910 (2005)

${ }^{8}$ J. Sese, J. Bartolome, and C. Rillo, Rev. Sci. Instrum. 78, 046101 (2007).

${ }^{9}$ See www.goodfellow.com/sp/ for reference CU045140.

${ }^{10}$ See www.alfa-chemcat.com for nickel slug $99.995 \%$, Puratronic (c).

${ }^{11}$ A. H. Morrish, The Physical Principles of Magnetism (Wiley, New York, 1965), p. 259.

${ }^{12}$ Quantum Design, MPMS AC Option User's Manual. 